\title{
A Relative Analysis of Genetic Algorithm and Binary Particle Swarm Optimization for Finding the Optimal Cost of Wind Power Generation in Tirumala Area of India
}

\author{
Prasun Bhattacharjee ${ }^{1, *}$, Rabin K. Jana ${ }^{2}$, and Somenath Bhattacharya ${ }^{1}$ \\ ${ }^{1}$ Department of Mechanical Engineering, Jadavpur University \\ ${ }^{2}$ Operations \& Quantitative Methods Area, Indian Institute of Management Raipur
}

\begin{abstract}
Although India presently holds the global fourth-biggest instated Wind Power Generation (WPG) capability, it necessitates advancing more rapidly to satisfy the rising energy requirement of its evolving economy while restraining the consequential greenhouse gas emission. To accomplish the impressive target of setting up 140 GW WPG competence by 2030 as proposed by the Government of India, a greater number of financially viable wind farms are required to function all over the country without further ado. This paper focuses on finding the optimal cost for WPG in the Tirumala area of Andhra Pradesh. Genetic Algorithm (GA) and Binary Particle Swarm Optimization (BPSO) have been employed concurrently with four randomly chosen terrain conditions. The research outcomes demonstrate the superior capability of BPSO to attain the most optimal cost of energy.
\end{abstract}

\section{Introduction}

The ceaseless release of $\mathrm{CO}_{2}$ with other greenhouse gases to the environment owing to diverse social activities is intensifying the mean air temperature and aberrant weather conditions which further initiate global climate change [1]. Renewable energy resources propose affluent alternates amid the mounting international apprehension for the restricted stock of fossil fuels and their hazardous penalties on the ecosystem [2]. Even during the Covid-19 pandemic in 2020, the usage of renewable energy grew by $3 \%$ while the requirement of every other fuel dropped universally [3].

Being the fourth-prominent power expending nation, India has an enormous capability to curb climate change. An ambitious goal of setting up the capability to generate $450 \mathrm{GW}$ from renewable energy resources by 2030 , including $140 \mathrm{GW}$ wind capacity, has been already communicated by the Central Government of India. Global Wind Energy Council (GWEC) Report for 2021 testifies that $39 \mathrm{GW}$ of wind power capacity could have been instated in India till February 2021. Being the second most populated nation on the Earth, it turns out to be enormously crucial for India to utilize more renewable resources to propel its developing economy in an ecofriendlier manner [4]. Globally, the cost of generating electricity from wind has shrunken sharply over the years [5]. That is why it becomes particularly essential for India to channelize added endeavors for meeting its incessantly mounting energy demand through wind power generation.

The National Institute of Wind Energy (NIWE) has already confirmed the wind power potential of $302 \mathrm{GW}$ at $100 \mathrm{~m}$ for India [6]. As of 31 January 2021, India retains $10.3 \%$ of its $377260.67 \mathrm{MW}$ total installed capacity from Wind Power Generation (WPG) units [7]. One of the most appealing features behind the prospect of wind power in India is that WPG the Levelized cost of electricity is almost 35\% lower than most of the coal-fired power generation units and this is likely to further falloff by $7 \%$ by 2022 [8]. The progress of WPG in India over the past five financial years has been displayed in Fig. 1.

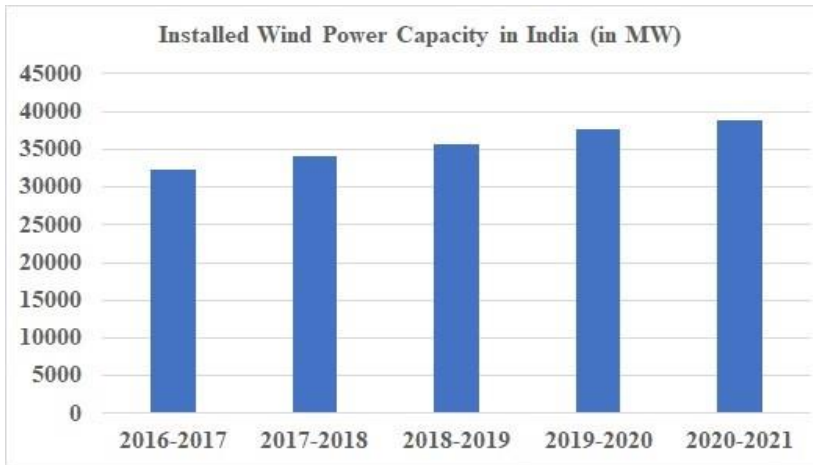

Fig. 1. WPG Capacity in India from F.Y. '16-'17 to '20-’21 [6]

\footnotetext{
*Corresponding author: prasunbhatta@gmail.com
} 
To check the upsurge of the global mean temperature much lower than 2 degrees Celsius as proposed by the Paris agreement of 2016 which has been ratified by 195 signatories including India, greenhouse gas emissions are needed to be cut through the rapid green energy transition. India, being principally a coal-dependent country, can achieve its emission cut goals and lower its fossil fuel dependency by identifying more profitable opportunities for WPG [9].

This paper focuses on identifying the optimal cost of WPG for the Tirumala area of Andhra Pradesh. NIWE has identified the WPG capacity of Andhra Pradesh as 44228.60 MW WPG of which 4077.37 MW could have been commissioned up to 31 October $2019[6,10]$. To find the optimal cost of WPG, several critical facets like terrain condition, wind velocity, and various cost components related to Wind Turbine (WT) are to be evaluated. As the wind flow over a region is inherently arbitrary, the optimal placement of WTs in a wind farm requires extensive computational effort to trade-off between the power output and the Cost of Energy (CoE). Artificial Intelligence (AI) techniques like Genetic Algorithm (GA) and Binary Particle Swarm Optimization (BPSO) have been engaged simultaneously to minimize the optimal $\mathrm{CoE}$ per unit electricity. The optimization outcomes from both AI-based methods are compared to evaluate their relative efficiency.

\section{Problem Formulation}

\subsection{Wind Power Calculation}

According to the theory of aerodynamics, the kinetic energy absorbed by the WT is calculated as per Eq. (1).

$$
P=\frac{1}{2} \rho A \vartheta^{3} C_{p} \cos \theta
$$

where $P$ is the kinetic energy accessible for extraction by WT, $\rho$ is the air density, $A$ is the wind wheel area, $v$ is the speed of the wind flow, $C_{p}$ denotes the power coefficient [as stated by the Betz's law, which is of the extreme value of 0.593 ] and $\theta$ is the error of yaw i.e., the angular deviation between the wind wheel revolution axis and the wind flow direction [11]. The wind energy obtainable for power generation for a solo WT positioned at $\left(\mathrm{x}_{\mathrm{i}}, \mathrm{y}_{\mathrm{i}}\right)$ has been expressed in Eq. (2).

$$
E=\int_{\theta} p(\theta) \int_{v} p_{v}^{\theta}\left(v ; c_{i}, k_{i} \mid \theta\right) \eta(v)
$$

where $p(\theta)$ denotes the possibility of airstream flow from directional angle $\theta, p_{v}{ }^{\theta}\left(v ; c_{i}, k_{i} \mid \theta\right) \eta(v)$ is the Weibull distribution. The energy captured by individual WTs is added to estimate the yield of the wind farm [12].

\subsection{Objective Function}

Wind farms are required to stay economically viable through proficiently handling the $\mathrm{CoE}$ which is calculated as per Eq. (3) which has been recommended in the $22^{\text {nd }}$
Genetic and Evolutionary Computation Conference held in 2015 [12].

$$
\begin{aligned}
& \operatorname{CoE}=\frac{\left(C_{t} N+C_{S} f \operatorname{loor}\left(\frac{N}{m}\right)\right)\left(\frac{2}{3}+\frac{1}{3} e^{-0.00174 N^{2}}\right)+\left(C_{o m} N\right)}{\left(1-(1+r)^{-y}\right) / r} * \frac{1}{8760 * P}+ \\
& \frac{0.1}{N}
\end{aligned}
$$

where $C_{t}$ signifies the expense due to a WT and is considered as USD 750000. $C_{s}$ characterizes the expenditure due to a sub-station and is considered as USD 8000000. $N$ indicates the count of WTs of the wind farm and $m$ indicates the number of WT per sub-station is considered as $30 . C_{o m}$ stands for the yearly operational and maintenance charge and is considered as USD 20000 per year. $r$ denotes the fraction of interest and is considered as $3 \% . y$ indicates the expected operational life of the wind farm and is considered as 20 years [12].

\subsection{Wind Flow Pattern and Terrain Condition}

In the current research work, Tirumala $\left(13^{\circ} 41^{\prime} 30.4^{\prime \prime} \mathrm{N}\right.$ $79^{\circ} 21^{\prime} 344^{\prime \prime}$ E) area of southern Indian state Andhra Pradesh has been selected for finding the optimal $\mathrm{CoE}$ for WPG [4]. The wind pattern from 2012 to 2017 as reported by Kumar et. al. has been displayed in Fig. 2 .

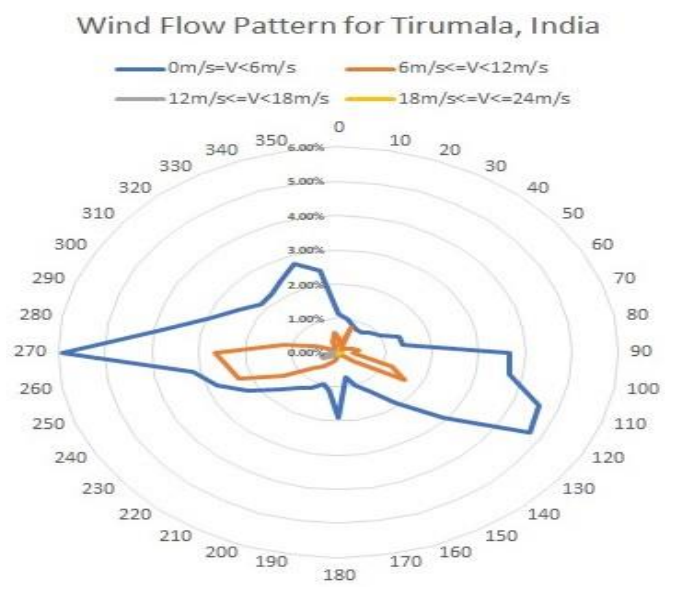

Fig. 2. Wind flow velocity distribution for Tirupati, India [4]

Four arbitrarily chosen terrain layouts have been considered for evaluation. Three layouts of them are with obstacles and the remaining one has no obstacles present in the wind flow path. The considered terrain conditions have been displayed in Figs. $3-6$.

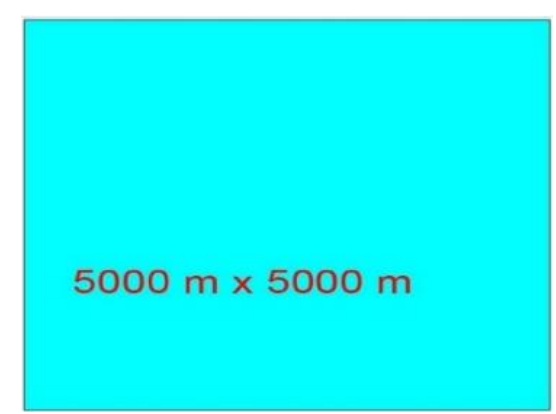

Fig. 3. Terrain condition 1 with no obstacles 


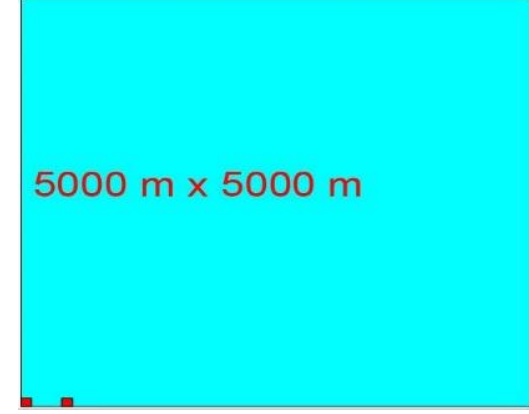

Fig. 4. Terrain condition 2 with two obstacles

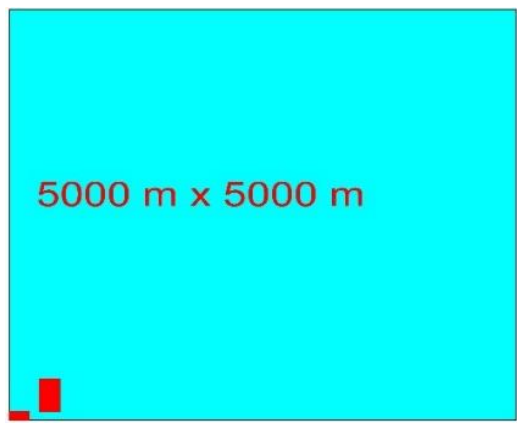

Fig. 5. Terrain condition 3 with two obstacles

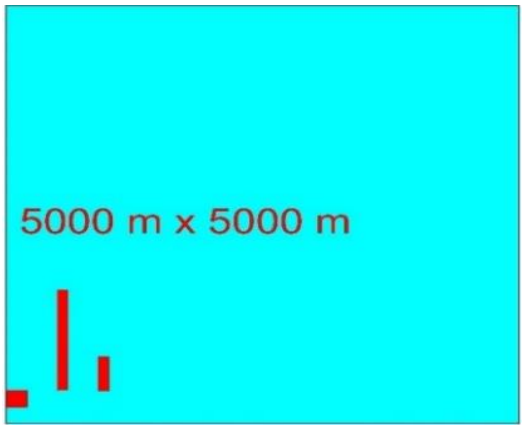

Fig. 6. Terrain condition 4 with three obstacles

\section{Optimization Algorithms}

In the present work. GA and BPSO have been engaged simultaneously to find the optimal $\mathrm{CoE}$ for the previously mentioned terrain conditions. GA is an AI-based search technique and has been extensively used in various engineering domains [13]. GA can be presented as follows.

1. Prepare the factors like population size, iteration count, chances for crossover, and mutation.

2. Set the population randomly.

3. Calculate the aptness of each chromosome.

4. Perform the arithmetic crossover process as follows:

4.1 Choose a number arbitrarily within 0 and 1. If it is less than the chance of crossover, choose the parental chromosome for the crossover method.

4.2 Instigate the crossover procedure.

4.3 Examine the viability of the offspring.

4.4 If the offspring is feasible, then integrate them into the recent population.
5. Complete the mutation method as follows:

5.1 Choose a number arbitrarily within 0 and

1. If it is less than the chance of mutation, pick the chromosome for the mutation method.

5.2 Institute the mutation procedure.

5.3 Validate the fresh chromosome.

5.4 If the created chromosome is feasible, integrate it into the recent population.

6. Test the aptness of the fresh entities created through crossover and mutation methods.

7. Pick out the most excellent result following the decision maker's predilection.

Particle Swarm Optimization (PSO) is another AIenabled searching methodology that emulates the social actions of bees through communicating the information related to the universal and limited finest solutions [14]. The BPSO is a variant of PSO that considers every particle as a bit string. The site of a 'particle' can be amended by exchanging between 0 and 1 consistent with the particle velocity [15]. For the $e^{\text {th }}$ bit of $f^{\text {th }}$ particle, the velocity $v_{e f}$ can be presented using Eq. (4)

$v_{e f}=w v_{e f}+c_{1} r_{1 f}\left(p_{e f}-x_{e f}\right)+c_{2} r_{2 f}\left(g_{f}-x_{e f}\right)$

where $w$ indicates the inertia weight and can be determined using Eq. (5).

$w=w_{\max }-\left(w_{\max }-w_{\min }\right) \frac{l}{l_{\max }}$

where $w_{\max }$ and $w_{\min }$ are the highest and lowest limits of inertia weight correspondingly. $l$ is the existing count of recurrence and $l_{\max }$ is the highest count of reiterations. $c_{1}$ and $c_{2}$ are acceleration parameters. $r_{l f}$ and $r_{2 f}$ are random variables ranging between 0 and $1 . p_{e f}$ specifies the $f^{\text {th }}$ bit of the distinct most excellent position of the $e^{\text {th }}$ particle. $g_{f}$ denotes the $f^{\text {th }}$ bit of the collective best position. The transfer function which is utilized to revise the bit value is formulated as per Eq. (6).

$s\left(v_{e f}\right)=\frac{1}{1+e^{-v_{e f}}}$

$x_{e f}= \begin{cases}1, & \text { if rand }() \leq s\left(v_{e f}\right) \\ 0, & \text { otherwise }\end{cases}$

where rand is a function that arbitrarily chooses a number with uniform distribution [15].

\section{Results and Discussion}

For assessing the relative performance of GA and BPSO, a similar objective function of minimization of $\mathrm{CoE}$ has been considered. CoE has been measured in USD $/ \mathrm{kW}$. Both the optimization techniques have been iterated 50 times. Population size has been considered as 20 for GA and BPSO. 1.5 MW WT of radius $38.5 \mathrm{~m}$ has been employed for the current research. To reduce the wake loss effect, the gap between two adjacent WTs has been kept as 8 times the WT radius. The cut-in and cut-off velocities for the considered WT are $3.5 \mathrm{~m} / \mathrm{s}$ and $20 \mathrm{~m} / \mathrm{s}$ 
respectively. The research outcomes have been shown in Fig. 7.

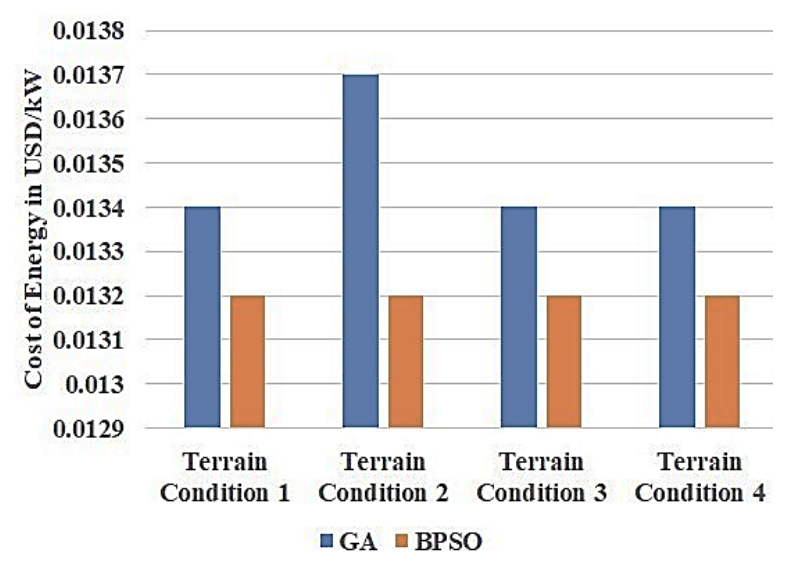

Fig. 7. Comparison of $\mathrm{CoE}$ for different terrain conditions

The plot displayed in Fig. 7 demonstrates that BPSO is more effective than GA for finding the optimal $\mathrm{CoE}$ for every terrain condition assumed with wind flow pattern for Tirumala. The BPSO algorithm is capable of finding CoE as low as USD $0.0132 / \mathrm{kW}$ consistently for every chosen terrain condition.

\section{Conclusion}

In the current paper, AI-based efficient techniques have been presented to find the optimal CoE for WPG in the Tirumala area of Andhra Pradesh state of India. GA and BPSO have been utilized concurrently to assess their relative effectiveness. The research outcomes prove the better suitability of BPSO in finding the optimal $\mathrm{CoE}$ for four randomly chosen terrain conditions. The present study will initiate novel opportunities for investigating the financial viability of WPG for different geographical locations worldwide.

The first author would like to express to the TEQIP department of Jadavpur University for providing a research grant for the current study.

\section{References}

1. B. Obama, Science 355 (6321), 126-129(2017)

2. P. K. Chaurasiya, V. Warudka, S. Ahmed, Energy Strategy Reviews 24, 342-357(2019)
3. https:/www.iea.org/articles/the-impact-of-thecovid-19-crisis-on-clean-energy-progress

4. M.B.H. Kumar, S. Balasubramaniyan, S. Padmanaban, J.B. Holm-Nielsen, Energies 12(11), 2158(2019)

5. R. Sitharthan, J.N. Swaminathan, T. Parthasarathy, 2018 National Power Engineering Conference (IEEE, Madurai, 2018)

6. https://en.wikipedia.org/wiki/Wind_power_in_India

7. https://cea.nic.in/renewable-generationreport/?lang=en

8. https://gwec.net/india-wind-outlook-towards-2022looking-beyond-headwinds/

9. https://www.downtoearth.org.in/blog/climatechange/the-world-is-warming-and-we-don-t-knowhow-much-carbon-can-it-take-67251

10. https://nredcap.in/PDFs/Tenders/RE_Status_31_10_ 2019.pdf

11. Z. Wu, H. Wang, Energy Procedia 16, $53-$ 57(2012)

12. D. Wilson, S. Rodrigues, C. Segura, I. Loshchilov, F. Hutter, G. L. Buenfil, A. Kheiri, E. Keedwell, M. Ocampo-Pineda, E. Özcan, S. I. V. Peña, B.

Goldman, S. B. Rionda, A. Hernández-Aguirre, K. Veeramachaneni, S. Cussat-Blanc, Renewable Energy 126, 681-691(2018)

13. R. K. Jana, P. Bhattacharjee, International Journal of Design Engineering 7(2), 77(2017)

14. A. Duggirala, R.K. Jana, R. V. Shesu, P. Bhattacharjee, (2018). Sādhanā 43(1), (2018)

15. J. Liu, Y. Mei, X. Li, IEEE Transactions on Evolutionary Computation 20(5), 666-681(2016) 